\title{
Behavioural consequences of nucleus accumbens dopaminergic stimulation and glutamatergic blocking in pigeons
}

\author{
Martín J. Acerbo $^{a}$, Pascual A. Gargiulo ${ }^{\mathrm{b}, *}$, Ines Krug a ${ }^{\mathrm{a}}$, Juan D. Delius ${ }^{\mathrm{a}}$ \\ a Allgemeine Psychologie, Universität Konstanz, 78457 Konstanz, Germany \\ ${ }^{\mathrm{b}}$ Laboratorio de Neurociencias y Psicología Experimental, Universidad Católica Argentina and Unidad de Neuroquímica y Farmacología del \\ Comportamiento, Universidad Nacional de Cuyo, 5500 Mendoza, Argentina
}

\begin{abstract}
Upon systemic administration of apomorphine, a potent dopamine agonist, pigeons show a bout of pecking behaviour. When the drug is repeatedly administered a sensitization takes place that is associated with pronounced discrimination learning. Here we show that intra-cerebral injections of apomorphine in the periphery of the nucleus accumbens of pigeons also elicit pecking. We additionally show that injections of 5-amino-phosphonohepatnoic acid, a NMDA-glutamate receptor blocker, into the Acc impairs the performance of a learned visual discrimination incorporating pecking as a choice response. We conclude that, as it is the case in mammals, the control mechanisms of learned sensory-motor behaviour in birds involves dopaminergic and glutamatergic synaptic transmission within the nucleus accumbens area.
\end{abstract}

Keywords: Pigeon; Nucleus accumbens; Pecking response; Learned discrimination; Glutamate antagonists; Dopamine agonist; Schizophrenia

\section{Introduction}

The nucleus accumbens septi (Acc) of the basal forebrain is a major component of the ventral striatum of mammals. Partly because of its probable involvement in the pathophysiology of human schizophrenia [13], it has received considerable attention over recent years. The mammalian Acc, among other things, appears to be involved in the learning and performance of sensorymotor behaviour [24,21]. Both dopaminergic and glutamatergic transmission appear to intervene in this Acc function [23,4]. Among several other inputs, the Acc does in fact receive dopaminergic projections from the ventro-tegmental area and glutamatergic projections from the cortico-limbic system [17].

Birds possess a structure that appears to be anatomically homologous to the Acc in mammals. As depicted in the standard pigeon brain atlas [14] it is located below the ventral edge of the lateral forebrain ventricles in the septal area. However, according to more recent findings

* Corresponding author. Fax: +54-261-428-7370

E-mail address: gargiulo@lab.cricyt.edu.ar (P.A. Gargiulo). the avian Acc appears to extends more ventrally, laterally, and posteriorly than delineated in the atlas, occupying a larger area surrounding the lateral, subventricular bed nucleus of the stria terminalis [25]. This general area receives dopaminergic input from the ventral mesencephalic tegmentum [9]. It is known that systemic administration of Apo has a rewarding effect in pigeons [6]. Previously we found that the neighborhood of Acc supports electrical self-stimulation responding in pigeons [7]. In mammals the delivery of brain reward is well known to be associated with the activation of dopaminergic mechanisms [30]. A glutamatergic innervation of the avian Acc has not yet been sought for, but it does receive corticostriatal-like projections [25]. In an earlier study in the course of which we proposed an animal model of schizophrenic delusional perceptions, we found that intra-Acc administration of a glutamate antagonist disrupted a learned visual discrimination task [11]. In view of the fact that injections of dopamine agonists, particularly apomorphine (Apo), into the Acc of mammals are known to stimulate oral motor activity [27], the present study sought to find out whether a similar stimulation would elicit pecking in pigeons. It is well established that in birds the systemic application of 
Apo elicits an intensive bout of pecking behaviour and that a sensitization that develops with repeated Apo administrations incorporates a marked visual discriminatory conditioning [15]. The study also sought to establish whether injections of further kinds of glutamate transmission inhibitors would similarly impair the performance of the earlier mentioned conditioned discrimination. Several different glutamatergic blockers have been found to depress the performance of learned behaviours in mammals $[2,5]$.

\section{Glutamatergic blocking}

\subsection{Method}

\subsubsection{Subjects}

Twenty adult domestic pigeons (Columba livia) of local homing stock and weighing between 450 and $550 \mathrm{~g}$ were used. They were kept in individual $40 \times 45 \times 35 \mathrm{~cm}$ stainless steel grid cages located in a well ventilated and illuminated (12-h light:12-h dark) room and were maintained at $80 \%$ of their normal weight throughout the experiment. All the experimental treatments described in this paper complied with the German animal protection laws and regulations.

\subsubsection{Apparatus}

Horizontal conditioning platforms controlled by a personal computer were used [11]. They were attached to the pigeons' home cages replacing their standard feeding troughs. Each platform incorporated two sideby-side transparent pecking keys (centers $5 \mathrm{~cm}$ apart, diameter $2.5 \mathrm{~cm}$ ). Two light-emitting-diode matrices $(5 \times 7$ green diodes, $12 \times 17 \mathrm{~mm})$ served to present visual patterns under these keys. Two solenoid feeders could deliver rewards consisting of a few grains of millet onto the keys.

\subsubsection{Procedure}

Twenty pigeons were first automatically shaped to peck the keys. This involved successive blocks of 40 trials. A trial began with a $20 \mathrm{~s}$ pause. A small pentagonal stimulus was then presented for $8 \mathrm{~s}$ randomly under either the right or left key, the other key remaining unlit. A peck to the illuminated key delivered an immediate reward into this key followed by a $2 \mathrm{~s}$ feeding time. However, if the animal did not peck, a reward was issued at the end of the stimulus presentation. As soon as $80 \%$ of the trials of a block yielded pecks, these latter free rewards were discontinued; the animals were only rewarded if they pecked the key during a now temporally unlimited presentation of the stimulus. When the subjects had emitted 40 such instrumental responses, they entered the training phase.
The daily training sessions consisted of 10 blocks of 40 trials each. A trial began with the simultaneous presentation of two discriminative stimuli under the keys. Two mirror-image visual stimuli were used, a plike shape and a q-like shape (size: $3 \times 5$ diodes, $7 \times 12$ $\mathrm{mm})$ in either a standard or an inverted $\left(180^{\circ}\right.$ rotated) orientation. The two types of stimulus pairs and the right/left key allocation of the relevant stimuli were randomized across the successive trials of each block. The $\mathrm{p}$ (or d) shape was defined as correct and the q (or b) shape as incorrect. Three pecks delivered to the key showing a correct stimulus yielded a reward accompanied by a $2 \mathrm{~s}$ feeding time with unlit matrices. Three pecks to the key displaying the incorrect stimulus yielded a penalty consisting of a $2 \mathrm{~s}$ time-out with the matrix fully illuminated. A $2 \mathrm{~s}$ inter-trial interval with dark matrices preceded the next trial. Trials ending in penalty were followed by a repeat trial with exactly the same stimulus configuration. This correction procedure ended when the correct stimulus was chosen. These repeat trials were separately recorded but were not included in the discrimination score calculations. The training phase ended when a criterion of $80 \%$ correct trials was achieved within a block of trials.

\subsubsection{Surgery}

The pigeons were then chronically cannulated. Anesthesia was induced by a combined xylazine and ketamine i.m. dose and maintained with additional doses of ketamine [1]. With a stereotaxic apparatus (Stoelting), each pigeon was implanted with two stainless steel guide cannulas (23 gauge, $13 \mathrm{~mm}$ long) with their beveled tips directed at the right and left Acc. The injection sites aimed for (see below) corresponded to the stereotactic coordinates A 8.5, L 2.0, D 7.5 of the pigeon brain atlas [14]. The cannulas were fixed to the skull with acrylic cement and were closed with removable stainless steel pins (30 gauge, $13 \mathrm{~mm}$ long). After surgery, the pigeons were allowed a week to recover before testing began.

\subsubsection{Testing}

Each test involved two sessions carried out on 2 consecutive days. Immediately before the first of these sessions the pigeons were i.c. injected bilaterally with 1 ul doses of saline and immediately before the second session they were injected bilaterally with $1 \mu \mathrm{g} 1$ doses of drug solution. The occlusion pin of the relevant cannula was removed and a 30-gauge stainless steel injection cannula connected to a microsyringe was inserted through the guide cannula. The length of this latter cannula $(15 \mathrm{~mm})$ was adjusted to reach the Acc at the above listed stereotactic coordinates. The volumes were gradually injected over 2-min periods and the cannula was left in place for an additional $1 \mathrm{~min}$ to allow diffusion [11]. The test sessions proceeded as described 
before, but consisted of three blocks of 40 reinforced trials each. Between the successive pairs of test sessions related to different drugs there was an interval of at least 2 days. The drugs (all supplied by Tocris) and doses used were $1 \mu \mathrm{g} / \mu 1$ 5-amino-phosphonohepatnoic acid (AP-5), $1 \quad \mu \mathrm{g} / \mathrm{ul}$ 2,3-dioxo-6-nitro-1,2,3,4-tetrahydro-benzo(f)quino-xaline-7-sulfonamide(NBQX), $1 \mu \mathrm{g} / \mu 1$ 1-aminocyclo-pentanecarboxilic acid (cycloleucine, Cyclo) and $1 \mu \mathrm{g} / \mu \mathrm{l}(5 \mathrm{R}, 10 \mathrm{~S})-(+) 5$-methyl-10,11-dihydro-5Hdi-benzo[a,d]cyclohepten-5,10-imine (dizocilpine, MK801). All drugs were dissolved in saline, in the case of AP-5 with the addition of $1 \% \mathrm{NaOH}$.

\subsubsection{Histology}

After the pigeons had completed this and the next experiment they were anaesthetized, i.c. injected with 1 $\mu$ Indian ink into each brain side and then perfused transcardially with saline and 4\% formaline solution. The brains were removed from the skull and postfixed in formaline solution. After having bathed in a $30 \%$ saccharose solution for a few days the brains were blocked and sectioned $(40 \mu \mathrm{m})$ with a cryotome. The tissue block was inspected with a $10 \times$ magnifying lens and every fifth section around the injection site was mounted. The injection sites were located with a microscope and transferred to standard brain section drawings.

\subsection{Results}

\subsubsection{Histology}

Of the pigeons that completed the experiment (see below) 14 pigeons had their injection sites (ink deposits, small lesions) bilaterally located in the Acc or its immediate periphery (Fig. 1). These pigeons formed the Acc group. In five pigeons at least one of the two injection sites lay outside the Acc or within the overlying lateral ventricle (ink deposits along the ventricular ependyma). These pigeons formed the Cntr group.

\subsubsection{Training}

One pigeon that did not reach the discrimination criterion within 75 sessions was excluded. The other 19 pigeons needed between two and 64 (median 11) sessions of discrimination training to reach the $80 \%$ correct criterion. In the subsequent saline test sessions, they maintained a mean performance of above $70 \%$ correct trials.

\subsubsection{Tests}

Direct observations indicated that the bilateral i.c. injection of AP-5 had neither motivational nor motor effects in so far as the pigeons key-pecked as accurately and fast as during saline sessions. However, the drug treatment lead an appreciable discrimination score impairment in the Acc group. The mean number of

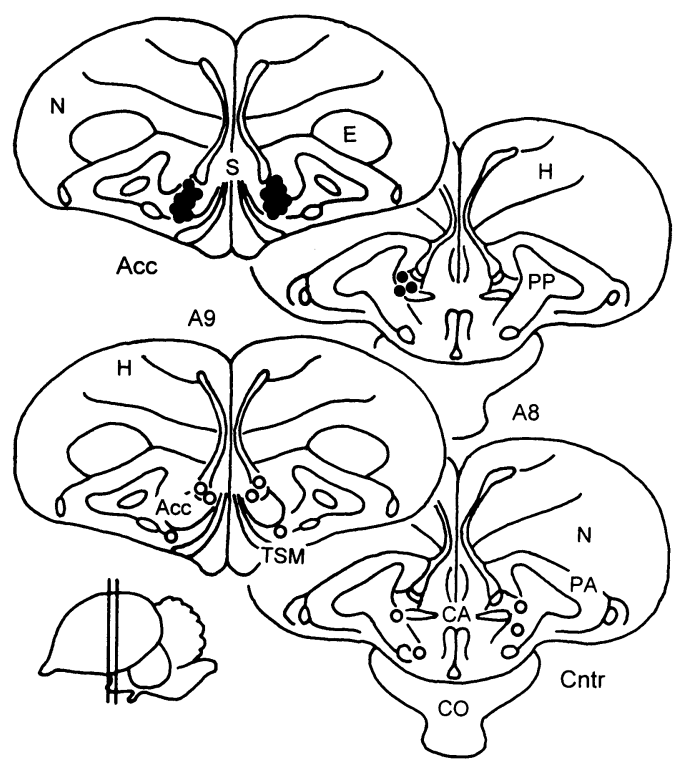

Fig. 1. Frontal brain sections showing the location of the glutamatergic drug injection sites. Injection sites of the 14 Acc group pigeons with both right and left sites (closed circles) within the Acc or its immediate neighborhood and of five Cntr group pigeons with one or both sites (open circles) outside the Acc (ventricle or otherwise). Abbreviations: Acc, nucleus accumbens; CA, comissura anterior; $\mathrm{CO}$, chiasma opticum; E, ectostriatum; $\mathrm{H}$, hyperstriatum; $\mathrm{N}$, neostriatum; PA, paleostriatum augmentatum; PP, paleostriatum primitivum; $\mathrm{S}$, septum: TSM, tractus septo-mesencephalicus. The labels A8 and A9 refer to stereotaxic planes.

correct trials over the three blocks of the drug test session dropped significantly compared with the corresponding score obtained during the preceding saline session (Table 1; Wilcoxon test, $T=12.0 n=14, P \approx$ $0.01)$ The mean number of correction trials in turn increased significantly $(T=14, n=14.5, P<0.05)$. The same dose of AP-5 injected i.c. into the Cntr group pigeons had no significant effect on performance in either respect ( $T s \geq 5, n=5, P>0.05$, Table 1, Fig. 2). It was apparent that the mere unilateral Acc injection of AP-5 that occurred in some of the latter birds was ineffective. The same had been previously found to be the case with AP-7 [11].

Bilateral NBQX, Cyclo and MK801 injections into the Acc (Acc group) had no significant effect on the discrimination performance as compared with the pre-

Table 1

Mean percentage of correct trials and number of correction trials $( \pm$ S.E.) after bilateral saline and 5-aminoheptanoic injections into the nucleus accumbens

\begin{tabular}{llllll}
\hline & \multicolumn{2}{l}{ Acc group $(n=14)$} & & \multicolumn{2}{c}{ Cntr group $(n=5)$} \\
\cline { 2 - 3 } \cline { 5 - 6 } & Saline & AP-5 & & Saline & AP-5 \\
\hline \% trials correct & $72.1 \pm 2.2$ & $65.7 \pm 2.5^{* *}$ & & $71.9 \pm 2.3$ & $71.6 \pm 2.6$ \\
Correction trials & $20.3 \pm 1.9$ & $26.5 \pm 3.2^{*}$ & & $16.7 \pm 2.0$ & $20.7 \pm 4.0$ \\
\hline
\end{tabular}

Asterisks indicate significant effects. 


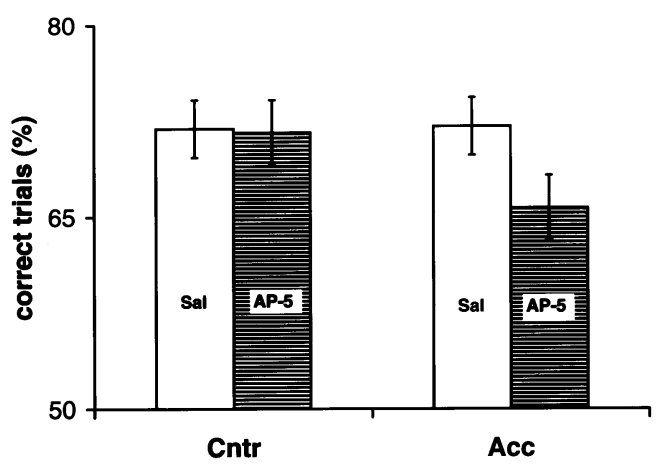

Fig. 2. Effects of 5-aminophosphonoheptanoic acid injected into the Acc on the discrimination performance and correction trials of pigeons. Means \pm S.E. of 14 Acc group and five Cntr group pigeons.

ceding saline baseline (Table 2). Even though a randomized treatment order would perhaps have been preferable it seems unlikely that the absence of impairing effects could be due to the fact that AP-5 injections might have somehow lesioned the Acc because during the subsequent saline injection sessions yielded an above $70 \%$ correct discrimination performance all through the experiment (Table 2). No-effect results were also obtained with NBQX, Cyclo and MK801 in the Cntr group but these are not tabulated.

\section{Dopaminergic stimulation}

\subsection{Methods}

\subsubsection{Subjects}

Of the 35 pigeons that initially took part in this experiment, 31 had participated either in the previous experiment or in a similar experiment involving the i.c. administration of other glutamate transmission blockers. All these pigeons had a break of at least 2 weeks before the present experiment began. The four additional pigeons were especially canulated for this experiment to ensure bilateral injection sites placed in the caudal paleostriatum (stereotaxic coordinates A 7.75, L 2, D 8.5;). All pigeons received ad libitum water and food in their home cages throughout this experiment.

\subsubsection{Apparatus}

A standard pigeon cage with the inner surfaces of its back and side walls lined with white cardboard panels randomly sprinkled with dark green dots of $0.8 \mathrm{~cm}$ diameter at a density of about 10 dots per $100 \mathrm{~cm}^{2}$ served as the experimental cage. The dots within the cage served as pecking targets [16].

3.1.2.1. Procedure. During a preparatory phase the pigeons were i.m. injected once daily on consecutive days with $0.5 \mathrm{mg} / \mathrm{kg}$ ready-made racemic Apo solution (Teclapharm), placed into the experimental cage, videorecorded for $20 \mathrm{~min}$ and then returned to their home cages. The preparatory treatment, normally extending to 4 days but in four pigeons for 6 additional days, served to generate a suitable sensitization to Apo and thus to increase the probability that the pigeons would show a pecking response to the subsequent i.c. Apo administrations. When this pretreatment was complete the pigeons were injected $20 \mu \mathrm{g} / \mu \mathrm{l}$ Apo levorotating (R(-)apomorphine-hydrochloride (supplied by ICN) dissolved in deoxygenated saline with $0.1 \%$ sodium disulfite as anti-oxidant) bilaterally into brain tissue. Nonracemic Apo was used to maximise the effective dose because saturation prevents the preparation of more concentrated Apo solutions [18,3]. These i.c. administrations were repeated on 3 consecutive days. After having been injected the pigeons were placed into the experimental cage and videorecorded for $20 \mathrm{~min}$.

3.1.2.2. Histology. After the experiment was completed the pigeons were sacrificed and their brains processed as already described under the previous experiment.

\subsection{Results}

\subsubsection{Histology}

Of the 28 birds which completed the experiment (see below), eight pigeons had both injection sites placed within the core of the Acc (Acor group), 11 pigeons had at least one injection site located in the ventro-lateral periphery of the Acc, that is, along the dorso-lateral edge of the tractus septo-mesencephalicus (TSM), the dorso-medial edge of the fasciculus prosencephali lateralis (FPL) and the dorso-lateral edge of the comissura anterior (CA; Aper group) and five pigeons that had at least one of the two injection sites located outside of the Acc or its periphery. These latter pigeons together with four pigeons with injection sites in the caudal paleostriatum primitivum (PP) formed the Cntr group (Fig. 3).

Table 2

Mean percentage of correct trials and number of correction trials $( \pm$ S.E. $)$ of the Acc group after saline, NBQX, Cyclo and MK801 treatments

\begin{tabular}{|c|c|c|c|c|c|c|}
\hline & Saline & NBQX & Saline & Cyclo & Saline & MK801 \\
\hline$\%$ corr. & $72.7 \pm 1.9$ & $71.2 \pm 2.1$ & $74.3 \pm 1.7$ & $75.7 \pm 1.9$ & $75.7 \pm 2.1$ & $75.7 \pm 2.0$ \\
\hline Corr. tr. & $17.8 \pm 1.6$ & $19.2 \pm 2.5$ & $14.8 \pm 1.2$ & $15.0 \pm 2.0$ & $13.9 \pm 1.2$ & $15.6 \pm 1.5$ \\
\hline
\end{tabular}

There were no significant effects. 


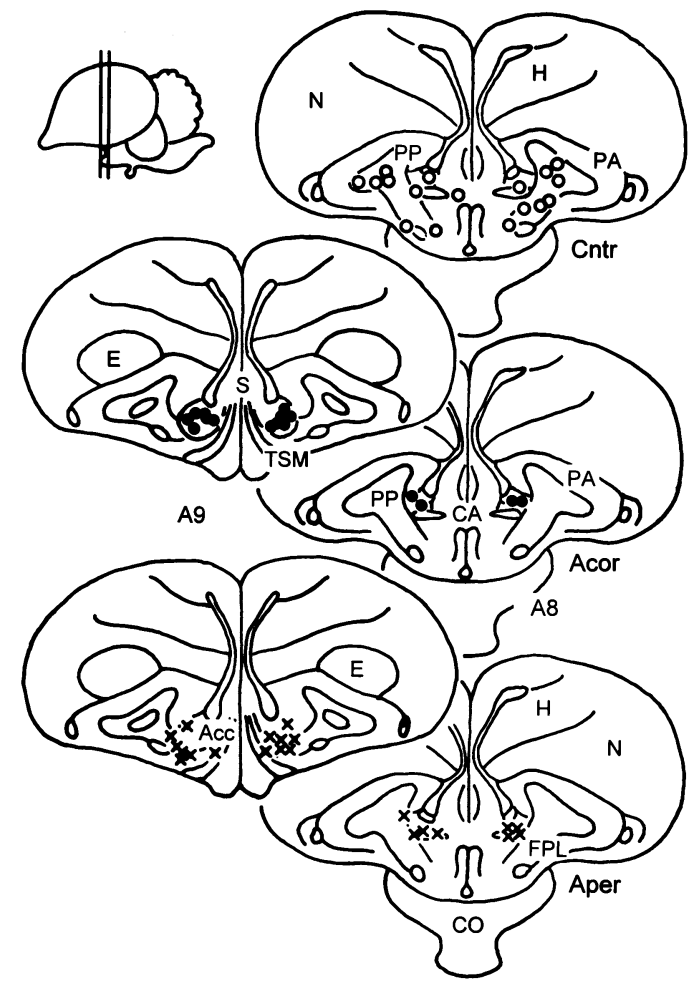

Fig. 3. Frontal brain sections showing the location of the Apo injection sites of the nine Cntr group pigeons (open circles), of the eight Acor group pigeons (closed circles) and the 11 Aper group pigeons (crosses). Same abbreviations as in Fig. 1 and fasciculus prosencepali lateralis, FPL.

\subsubsection{Behaviour}

During the preparatory phase, 28 pigeons evinced a bout of pecking upon the first $0.5 \mathrm{mg} / \mathrm{kg}$ i.m. dose of Apo and showed an increasing response to the repeated applications of the same dose. The seven pigeons which did not peck or pecked little during this preparatory phase were excluded from the experiment. Individuals that are unresponsive to Apo for genetic reasons occasionally turn up in our breeding stock. It was patent that in agreement with previous findings [16] all the remaining pigeons underwent a sensitization to Apo up to a nearly asymptotic level of responding of about 3000 pecks per 20 min. Fig. 4, left, shows the mean pecking scores of the responsive pigeons during the preparatory phase. There were no significant differences between the Aper, Acor and Cntr groups concerning the pecking rates during the final sensitization session (Kruskal-Wallis, $H=2.26, d f=2, n=28, P>0.05$ ).

Fig. 4, right, shows the mean pecking scores of the three pigeon groups during the i.c. Apo administration phase. The pigeons of the Aper group exhibited initial pecking responses that, although clearly less intensive than those shown previously in response to i.m. Apo injections, was still significantly above those shown by the Cntr group (Mann-Whitney tests; session five, $U=$ 13, $P<0.01$; session $6, U=21, P<0.05$; session seven,

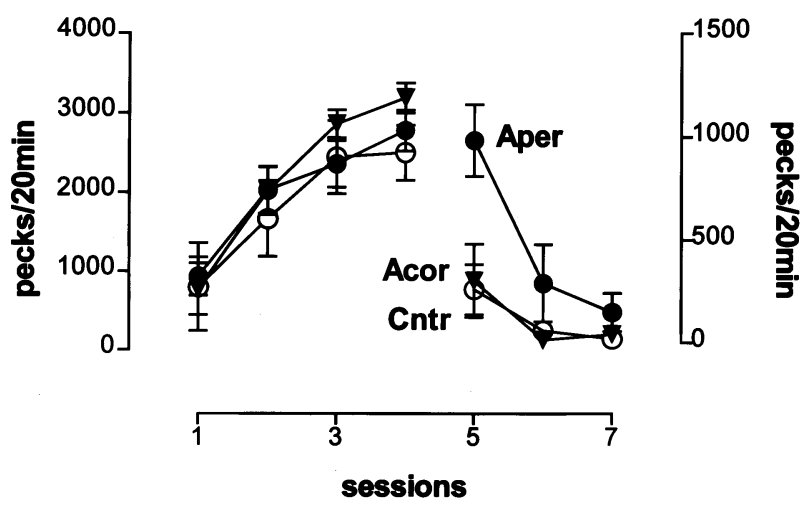

Fig. 4. Left: mean pecking scores \pm S.E. of the Aper, Acor and Cntr groups of pigeons during the i.m. Apo sensitization phase. Right: mean pecking scores \pm S.E. of the same Aper, Acor and Cntr groups while being i.c. injected with Apo. Note the different scales corresponding to the right and left sections of the graph.

$U=39.5, P>0.05 ;$ all $n_{1}=9$ and $\left.n_{2}=11\right)$. The Acor group somewhat surprisingly exhibited pecking responses that were throughout indistinguishable from those shown by the Cntr group. The response to the i.c. applications of Apo decayed with the successive treatments. In the case of the Aper group this decay was significant (session five $>$ session seven, Wilcoxon Test, $T=1.0, n=11, P<0.005$ ). Injections directly into the core of the Acc yielded a significantly weaker pecking response than injections into the periphery of the Acc (Mann Whitney tests, session five and six, $U \mathrm{~s}>8, n_{1}=8$ and $n_{2}=11, P<0.01$ ). The responses of the Acor group did not statistically differ from that of the Cntr group.

\section{Discussion}

\subsection{Glutamatergic blocking}

AP-5 injections bilaterally into the Acc or in its vicinity had a transitory but significantly detrimental effect on the pigeons' performance of a learned visual shape discrimination task. This treatment led to nearly chance levels of stimulus choice. The accompanying increased number of correction trials may be indicative of a stereotyped choice persistence [20]. Earlier, Gargiulo et al. [11] had shown that i.c. applications of 7amino-phosphonohepatnoic acid (AP-7) into the Acc of pigeons had an analogous impairing effect on the performance of the same visual discrimination task.

Since in mammals AP-5 is known to be an NMDA glutamate receptor blocker with a very similar action profile as AP-7 [26], which has a marked effect on the Acc functioning in rats [22], it is now practically certain that the Acc of pigeons, like that of mammals, incorporates glutamatergic synapses. Peculiarly, only NMDA and not AMPA or KA glutamate receptors appear to be implicated in the discrimination task 
employed here as NBQX, a non-NMDA receptor blocker, did not have a detrimental effect. Furthermore, the fact that also Cyclo, a blocker of the glycine allosteric site of the NMDA receptor and MK801, a blocker of the ion channel coupled to the NMDA receptor, also had no detrimental effect on discriminative performance suggest that the avian NMDA receptors might have a somewhat lower affinity for these drugs than those of mammals. It is possible that higher doses of these drugs might still prove effective. MK 801 has in fact been found to have a blocking effect on the conditioning connected with the sensitization to Apo mentioned earlier (Acerbo and Delius, in preparation). In rats the NMDA blockers disrupt both the acquisition [10] and the performance of learned behaviours [22]. Non-NMDA blockers appear to have a somewhat different but still important disruptive effect on cognitive processes $[8,21]$.

\subsection{Dopaminergic stimulation}

Apo is a potent dopaminergic agonist which acts on both the D1 and the D2 type of dopamine receptors [3]. We could confirm that systemically administered Apo elicited a sustained bout of stereotyped pecking and that repeated Apo administrations lead to an enhancement of that response. From previous studies we know that the sensitized pecking is importantly determined by learning [16] and that it in fact leads to visually discriminative responding [15]. This is undoubtedly related to the circumstance that systemically administered Apo has a rewarding effect [6].

Previous studies have furthermore indicated that i.c. injections of Apo into various other brain structures of the pigeon can also elicit pecking $[12,18]$. However, so far only the Apo induced pecking obtainable from the nucleus basalis prosencephali has been documented with adequate detail $[18,29]$. The present second experiment showed that injections of Apo into the periphery of the Acc elicited comparatively stronger pecking responses. Nevertheless, it is notable that upon repeated injections there was a response decay much as that reported by Lindenblatt and Delius [18]. These decays might possibly be due to the development of some local tolerance to Apo. But what is certain is that in the present experiment Apo injected into the central core of the Acc did not induce a sizeable pecking response. This suggests that the core and shell of the Acc of birds may not be equivalent in function, much as this has indeed been found in mammals [19]. Regarding the glutamatergic blockers AP-5 (present study) and AP-7 [11] they appear to act in the core and its closer surround but not in the outer periphery of the Acc. Unfortunately there is not as yet any anatomical or lesion data available that would provide a more definite basis for dividing up the avian Acc region.
Interestingly, Apo injected into the caudal paleostriatum did not elicit a significant pecking reaction although the earlier cited studies mention some paleostriatal injection sites positive for pecking. However, the paleostriata of pigeons are large structures likely to be parcellated into several functionally distinct areas. Only a small portion of these structures was sampled here.

According to a model proposed by Wickens [28,23], the coincidental activation of dopaminergic and glutamatergic afferences to striatal neurons leads to lasting alterations of glutamatergic synapses and thus to learned modifications of sensory-motor coordinations. We find that glutamatergic transmission within the Acc of pigeons is involved in the performance of a visual discrimination in pigeons and that dopaminergic transmission within the immediate periphery of the same nucleus is involved in the production of pecking, a motor response that coincides with that controlled by the said discrimination. It seems possible that the avian Acc, as part of their ventral striatum, is a substrate of sensory-motor learning that functions along the same principles conceived by Wickens [28] with the striatum of mammals in mind.

\section{Acknowledgements}

The research was supported by a cooperation grant of the Volkswagen Stiftung, Hannover, to P.A. Gargiulo and J.D. Delius and by a research grant of the Deutsche Forschungsgemeinschaft, Bonn, to J.D. Delius. We thank Anke McLintock for help with manuscript preparation and Jennifer Lee for correction of the English text.

\section{References}

[1] Aldavert-Vera L, Costa-Miserachs DM, Divac I, Delius JD. Presumed 'prefrontal cortex' lesions in pigeons: effects on visual discrimination performance. Behav Brain Res 1999;102:165-70.

[2] Aultman JM, Moghaddam B. Distinct contributions of glutamate and dopamine receptors to temporal aspects of rodent working memory using a clinical relevant task. Psychopharmacology 2001;153:353-64

[3] Baldessarini RJ, Kula NS, Zong R, Neumeyer JL. Receptor affinities of apomorphine enantiomers in rat brain tissue. Eur $\mathbf{J}$ Pharmacol 1994;254:199-203.

[4] Baldwin AE, Sadeghian K, Holahan MR, Kelley AE. Appetitive instrumental learning is impaired by inhibition of cAMP-dependent protein kinase within the nucleus accumbens. Neurobiol Learn Mem 2002;77:44-62.

[5] Baron SP, Moerschbaecher JMJ. Disruption of learning by excitatory amino acid receptor antagonists. Behav Pharamacol 1996;7:573-84.

[6] Burg B, Haase C, Lindemblatt U, Delius JD. Sensitization to and conditioning with apomorphine in pigeons. Pharmacol Biochem Behav 1989;34:59-64. 
[7] Delius JD, Pellander K. Hunger dependence of electrical brain self-stimulation in the pigeon. Physiol Behav 1982;28:63-6.

[8] Di Ciano P, Cardinal RN, Cowell RA, Little SJ, Everitt BJ. Differential involvement of NMDA, AMPA/kainate, and dopamine receptors in the nucleus accumbens core in the acquisition and performance of pavlovian approach behavior. J Neurosci 2001;21:9471-7.

[9] Durstewitz D, Kröner S, Hemmings HC, Güntürkün O. The dopaminergic inervation of the pigeon telencephalon: Distribution of the DARP-32 and co-ocurrence with glutamate decarboxylase and tyrosin hydroxylase. Neuroscience 1998;83:763-79.

[10] Gargiulo PA, Martínez G, Ropero C, Funes A, Landa AI. NMDA glutamatergic blockade of nucleus accumbens disrupts acquisition but not consolidation in a passive avoidance task. Ann NY Acad Sci 1999;877:717-22.

[11] Gargiulo PA, Siemann M, Delius JD. Visual discrimination in pigeons impaired by glutamatergic blockade of nucleus accumbens. Physiol Behav 1998;63:705-9.

[12] Goodman I. Amphetamine and apomorphine induced stereotyped behavior in adult pigeons. Pharmacol Biochem Behav 1981;15:701-4.

[13] Gray JA. Dopamine release in the nucleus accumbens: the perspective from aberrations of consciousness in schizophrenia. Neuropsychology 1995;33:1143-53.

[14] Karten HJ, Hodos WA. Stereotaxic atlas of the brain of the pigeon. Baltimore: Hopkins, 1967.

[15] Keller S, Delius JD. Discriminative learning occasioned by the administration of a dopamine agonist. Psychopharmacology 2001;157:320-3.

[16] Keller S, Delius JD, Acerbo MJ. Apomorphine sensitization: evoking conditions, context dependence, effect persistence and conditioned nature. Behav Phamacol 2002;13:1-14.

[17] Koob GF. Neural mechanisms of drug reinforcement. Ann NY Acad Sci 1992;654:171-91.

[18] Lindenblatt U, Delius JD. Nucleus basalis prosencephali, a substrate of apomorphine-induced pecking in pigeons. Brain Res 1988;453:1-8.

[19] Meredith GE, Totterdal S. Microcircuits in nucleus accumbens' shell and core involved in cognition and reward. Psychobiology 1999;27:165-86.

[20] Robbins TW. Cognitive deficits in schizophrenia and Parkinson's disease: neural basis and the role of dopamine. In: Willner P,
Scheel-Kruger J, editors. The mesolimbic dopamine system: from motivation to action. New York: Wiley, 1991:497-528.

[21] Roullet P, Sargolini F, Oliverio A, Mele A. NMDA and AMPA antagonist infusions into the ventral striatum impair different steps of spatial information processing in a nonassociative task in mice. J Neurosci 2001;21:2143-9.

[22] Scheel-Kruger J, Willner P. The mesolimbic system: principles of operation. In: Willner P, Scheel-Kruger J, editors. The mesolimbic dopamine system: from motivation to action. New York: Wiley, 1991:559-97.

[23] Smith-Roe SL, Kelley AE. Coincident activation of NMDA and dopamine D1 receptors within the nucleus accumbens core is required for appetitive instrumental learning. $\mathrm{J}$ Neurosci 2000;20:7737-42.

[24] Tzschentke TM, Schmidt WJ. Functional relationship among medial prefrontal cortex, nucleus accumbens, and ventral tegmental area in locomotion and reward. Crit Rev Neurobiol 2000;14:131-42.

[25] Veenman CL, Wild JL, Reiner A. Organization of the avian 'corticostriatal' projection system, a retrograde and anterograde pathway tracing study in pigeons. J Comp Neurol 1995;354:87126.

[26] Viana MR, Izquierdo LA, Barros DM, De Souza MM, Rodrigues C, Sant'Anna MK, Medina JH, Izquierdo I. Pharmacological differences between memory consolidation of habituation to an open field and inhibitory avoidance learning. Braz J Med Biol Res 2001;34:233-40.

[27] Voikar V, Soosaar A, Volke V, Koks S, Bourin M, Mannisto PT, Vasar E. Apomorphine-induced behavioural sensitization in rats: individual differences, role of dopamine and NMDA receptors. Eur Neuropsychopharm 1999;9:507-14.

[28] Wickens J. Striatal dopamine in motor activation and rewardmediated learning: steps towards a unifying model. J Neural Transm 1990;80:9-31.

[29] Wynne B, Delius JD. Frontal forebrain lesions: effects on the foraging and apomorphine pecking of pigeons. Physiol Behav 1996;59:757-62.

[30] Yavich L, Tiihonen J. In vivo voltammetry with removable carbon fibre electrodes in freely-moving mice: dopamine release during intracranial self-administration. J Neurosci Methods 2000;104:55-63. 